\title{
LAS COLECCIONES DE CUENTOS INFANTILES DE FERNÁN CABALLERO Y DE LOS HERMANOS GRIMM
}

\author{
THE CHILDREN'S TALE COLLECTION BY FERNÁN CABALLERO AND BY THE \\ GRIMM BROTHERS \\ SIMONE MANN ${ }^{1}$ \\ M.A. Cultura y Pensamiento Europeo: Proyección y Pervivencia \\ Universidad de León y Universidad de Bonn (Alemania)
}

\begin{abstract}
Resumen
Varios cuentos españoles recogidos y publicados por Fernán Caballero poseen el mismo contenido o la misma estructura que los cuentos alemanes recogidos por los hermanos Grimm. En este artículo comentamos las razones, las relaciones históricas de España y Alemania en aquella época, las influencias literarias de otros países como Francia y los elementos tomados de la cultura popular, a partir de leyendas y mitos, de lugares o seres maravillosos que a lo largo de los siglos siempre han existido. A partir de estas preguntas relacionaremos las características comunes con el hecho de la aparición de diversos cuentos en varios países. Por otra parte los cuentos ejercen una importante función psicológica para niños porque expresan sentimientos humanos universales, independientes de la época y cultura.
\end{abstract}

Palabras clave: Fernán Caballero, Hermanos Grimm, narrativa, cuento literario, Romanticismo.

\begin{abstract}
A lot of Spanish fairytales collected and published by Fernán Caballero have the same content or the same structure as the German fairytales collected by the Brothers Grimm. In this article we will comment the reasons, the historic relationship between Spain and Germany during that epoch, the literal influences of other countries like France and the elements taken from popular culture, through legends and myths, marvelous places or beings which have always existed through all the centuries. All these questions will help to connect the common characteristics with the appearance of various fairytales in different countries. Another fact is that the fairytales perform an important psychological function for children because they deal with universal human feelings, regardless of the epochs and cultures.
\end{abstract}

Key words: Fernán Caballero, Brother Grimm, Narrative, European tales, Romanticism.

Érase una vez una escritora, con una madre medio irlandesa y medio española y con un padre alemán, que se llamó Cecilia Böhl de Faber y que publicó la colección de cuentos infantiles Cuentos de encantamiento en 1877 bajo el pseudónimo Fernán Caballero, y dos hermanos alemanes con gran influencia en el mundo literario, los hermanos Grimm que publicaron sus Kinder- und Hausmärchen en tres volúmenes a partir del año 1812. En estas dos colecciones existen varios cuentos que se parecen

\footnotetext{
${ }^{1}$ Universidad de Bonn. simone4490@aol.com. Recibido: 28-04-2016. Aceptado: 16-07-08-2016.
} 
bastante en relación a su estructura y contenido. Por eso resulta interesante examinar las razones para la coincidencia. ¿Es explicable por las relaciones históricas entre España y Alemania como lo dijeron los propios hermanos Grimm (Grimm 1980: $424)$, por un mismo origen en otro país, por las influencias literarias de Francia o por pertenecer a la cultura popular? $\mathrm{O}$ hay que atribuir la coincidencia al padre de Cecilia, Nicolás Böhl de Faber (1770-1836) introductor del romanticismo en España, y gracias al cual Cecilia conocía bien la cultura, la literatura, y los teóricos alemanes como los hermanos Friedrich y August Wilhelm Schlegel (1772-1829 y 1767-1845) o Johann Gottfried Herder (1744-1803) (Herrero 1963: 68 y 107)?

Como es sabido, el género del cuento experimentó un auge durante la época literaria del romanticismo, sin embargo ya antes y asimismo en las épocas literarias siguientes lo popular siempre tenía gran valor ${ }^{2}$. En aquella época del romanticismo, se prestó especial atención a las raíces culturales de cada pueblo y a la importancia de las tradiciones populares como lo hicieron del mismo modo Fernán Caballero y los hermanos Grimm mediante la recolección y publicación de "sus" cuentos 3 .

\footnotetext{
${ }^{2}$ Ya en 1697, Charles Perrault publicó su colección Contes du temps passé avec des Moralités o sea Les contes de ma mère l'Oye que se convirtió junto con el Pentamerone de Giambattista Basile de los años 1634-1636 en una de las fuentes más importantes de cuentos (prólogo Grimm 1986a: XVIs., Rölleke 1985: 14 y Basile 1954: 502). Debido a las relaciones que Cecilia mantuvo con Francia - fue educada por una anciana belga y en un colegio administrado por monjas francesas (Herrero 1963: 47s. y Klibbe 1973: 15) - seguramente conoció la influencia de las obras de las coleccionistas francesas Madame d'Aulnoy (Contes de fées, 1697) y Jeanne-Marie Leprince de Beaumont (Le magasin des enfants, 1757) (Amores 2001: 140). La palabra alemana "Naturpoesie" (poesía natural) - de la que forma parte el cuento popular - fue introducida en la crítica y teoría literaria por el teórico alemán Johann Gottfried Herder (1744-1803). Fue él quien empezó a partir del año 1796 a recoger canciones populares y, especialmente, cuentos infantiles (Neumann 1986: 57 y Pöge-Alder ${ }^{22011: 123 s s .) ~ a u ́ n ~ a n t e s ~ d e ~ l o s ~}$ hermanos Grimm, que comenzaron a buscar y publicar obras de la tradición oral alemana a partir del año 1806. No obstante, algunas de estas narraciones son versiones de relatos ya existentes en otros países. Por ejemplo, algunas provienen de cuentos de Charles Perrault (Gerstner 1952: 48 y Rölleke 1985: 14). Además, existen motivos en los cuentos que provienen de otros géneros literarios como por ejemplo de las fábulas, de los poemas o de los lais. Un ejemplo de una obra en la que están indicadas algunas fuentes de cuentos existe con la colección de anotaciones a todos los cuentos de los hermanos Grimm (Anmerkungen zu den Kinder- und Hausmärchen der Brüder Grimm, 1913-1932) de Johannes Bolte y Georg Polívka. Siguiendo el ejemplo de los hermanos Grimm, se publicaron del mismo modo colecciones con relatos populares en los otros países europeos (Baquero Goyanes 1992: 2).

${ }^{3}$ Ciertamente, todos los tipos de cuentos comparten elementos generales, como por ejemplo las fórmulas introductorias ("Érase una vez", "Es war einmal") y de cierre ("Colorín, colorado, este cuento se ha acabado", "und wenn sie nicht gestorben sind, dann leben sie noch heute") (Morote Magán 2002: 176-184). Otros aspectos generales del género del cuento son el juego con colores y números, el tiempo y el lugar indeterminados (con un centro en el que la trama se desarrolla) y las referencias a la realidad extratextual con elementos de tiempos pasados y actuales (Faivre 1979: 29-32, Lüthi 2004 $4^{10}$ : 115, Morote Magán 2002: 162, Röhrich 19743: 202 y 242 y Von Beit 1952: 21s.). Además del elemento maravilloso y sobrenatural y de la descripción de la naturaleza (Hamann 1970: 122), se muestra típica una figura simple (del pueblo o de la aristocracia) y arquetípica que no envejece (Morote Magán 2002: 176-181 y Faivre 1979: 29-32). Por último, siempre se trata de dos figuras extremas en la tradición oral (e.g. tonto-listo, bueno-malo, guapo-feo); una figura está caracterizada por sus virtudes y una figura está caracterizada por sus vicios (Morote Magán 2002: 176-181). En conclusión, las dos colecciones naturalmente pueden parecerse, pero aparte de todos estos elementos generales, es posible constatar aún más coincidencias, lo que hace una comparación exacta imprescindible.
} 
Fernán Caballero es una "pionera" en la recogida de relatos populares españoles (Amores 2001: 8) orientándose no solamente a los hermanos Grimm - en cuanto a recoger textos de la tradición oral se refiere - sino asimismo en cuanto a mantener la versión más fiel al original en la medida de lo posible según el concepto de "fidelidad y verdad" (Amores 2001: 60). Se necesita este concepto para mostrar estas formas perfectas por las que se destaca la poesía natural, es decir, la popular según Jacob Grimm. Los contenidos de cualquier expresión de la poesía natural deben mantener el espíritu puro de la temprana poesía épica. No es posible de imitarla, sale del colectivo y por eso resulta - al contrario de la poesía artificial que proviene de un individuo de incalculable valor (Rölleke 1985: 61, Neumann 1986: 57, Morote Magán 2002: 162 y Amores 2001: 56). En sus conferencias, A. W. Schlegel habló por ejemplo sobre la historia de la literatura romántica (Schlegel: 1965) y comunicó sus ideas respecto a la poesía natural que se caracterizaba por el heroísmo, el amor, el honor y la religión (Schlegel 1966: 24s.). Lógicamente, es posible de encontrar estos cuatro componentes en el cuento popular.

El análisis de las fuentes e influencias franceses e italianas se propone en adelante a partir de la comparación de ocho parejas de cuentos, tomados de Cuentos de encantamiento de la escritora española y sus equivalentes alemanes de los Kinderund Hausmärchen ${ }^{4}$. En todos los casos, de la atención a los cuentos de las primeras ediciones se espera una mayor cercanía al relato folclórico original ${ }^{5}$. Además, había que distinguir entre cuentos de encantamiento y cuentos de animales.

Las parejas mejor estudiadas en el presente artículo son: "El lobo bobo y la zorra astuta" de Fernán Caballero y "Katz' und Maus in Gesellschaft" de los hermanos Grimm, que están definidos como cuentos de animales, y "El lirio azul" y "Der singende Knochen" como ejemplo de cuentos de encantamiento. Como fundamentos teóricos para esta comparación adoptamos el método de examen de Vladimir Propp (Morphologie du conte, 1970) y la teoría psicológica de Bruno Bettelheim (The Uses of Enchantment. The Meaning and Importance of Fairy Tales, 1976). Otro teórico importante es Stith Thompson quien publicó un índex con los motivos típicos del folclore en seis volúmenes (Motif-Index of Folk-Literature, 1955-1958). En el presente artículo no tratamos los motivos de los cuentos analizados detalladamente, pero seguramente se reconoce algunos por otros cuentos $u$ obras.

Aunque Vladimir Propp analizó para su teoría solamente cuentos de encantamiento, sugiere que su teoría es adecuada para desmontar de manera semejante otros tipos de cuentos, y a modo de ejemplo, los cuentos de animales (Propp 1970: $33,127,123)$. En realidad, en varios casos hay que ampliar o modificar su método de

\footnotetext{
${ }^{4}$ Estas parejas son: "Bella-Flor" y "Ferenand getrü un Ferenand ungetrü", "El lirio azul" y "Der singende Knochen", "El duendecillo fraile" y "Von den Wichtelmännern", "Los deseos" y "Der Arme und der Reiche", "La hormiguita" y "Läuschen und Flöhchen", "El Carlanco" y "Der Wolf und die sieben jungen Geißlein", "Benibaire" y "Herr Korbes" y por último, "El lobo bobo y la zorra astuta" y "Katz' und Maus in Gesellschaft".

${ }^{5}$ Véase Grimm 1986a y 1986b y Caballero 1878. La escritura de los ejemplos del texto no está adaptada a la costumbre ortográfica actual.
} 
análisis para poder adaptar las funciones a un cuento de otro tipo. Algunos critican la teoría de Vladimir Propp porque no indica cuántas funciones y esferas de acción son necesarias como mínimo, o consideran falsas las tesis de Vladimir Propp (Lüthi ${ }^{10}$ 2004: 122 y Nathhorst 1969: 28). Se refiere a la tercera tesis que indica que la orden de las funciones es siempre la misma (Propp 1970: 32), una tesis que Vladimir Propp mismo rebate en otro capítulo de su obra (Propp 1970: 132s.) y a la cuarta tesis que todos los cuentos de encantamiento pertenecen, debido a su estructura, al mismo tipo (Propp 1970: 33). Bertel Nathhorst critica, por ejemplo, que la función VIIIa no resulta realmente una función teniendo en consideración que Propp define las funciones como acciones de una figura y la carencia no describe una acción (Nathhorst 1969: 1629). En resumen, critica que "Propp's proposal is as a whole vague, his discussion of it contains contradictions, and his practice does not coincide with his theory" (Nathhorst 1969: 20).

Sin embargo, el método resulta muy apropiado en este caso, porque ofrece un sistema detallado para estructurar los cuentos y dividirlos en las partes fundamentales ${ }^{6}$. Otros teóricos como por ejemplo André Jolles (Einfache Formen, 1930) se dedicaron del mismo modo al género del cuento, pero sus obras explican más bien los diferentes relatos de la tradición oral en general, y de esa manera no sirven para un análisis en detalle de dos cuentos. Gérard Genette publicó por ejemplo la obra Discours du

\footnotetext{
${ }^{6}$ En este artículo no va a tratado en detalle la teoría de Vladimir Propp, sino presuponemos que está sabida. Las funciones relevantes para el análisis son las siguientes:

I alejamiento temporal de la casa por uno de los miembros de la familia, $\beta$.

$\mathrm{V} \quad$ información sobre el víctima obtenida por el agresor, $\zeta$.

VI engaño al héroe por el agresor para apoderarse del víctima o de un objeto, $\eta$

VII complicidad del víctima dejándose engañar y ayudando de esa manera al agresor, $\theta$.

VIII daño, el agresor da perjuicio a uno de los miembros de la familia, A.

VIII carencia, a uno de los miembros de la familia falta algo el miembro pretende poseer algo.

IX mediación, momento de transición, la noticia del daño o de la carencia está divulgada, alguien se dirige al héroe con una ruega o un orden, se manda o deja de salir al héroe, B.

X principio de la acción opuesta, el héroe en búsqueda acepta el orden o se decide a actuar, C.

XI partida, el héroe sale de la cada, $\uparrow$.

XIII reacción del héroe a la acción del futuro donante, E.

XIV recepción del objeto mágico por el héroe, $\mathrm{F}$.

XV desplazamiento entre dos reinos, viaje guiado del héroe al lugar del objeto, G.

XVI combate entre el héroe y el agresor, $\mathrm{H}$.

XVII marca, el héroe recibe una marca, I.

XVIII victoria sobre el agresor, $\mathrm{J}$.

XIX reparación del perjuicio inicial o de la carencia, $\mathrm{K}$.

XX vuelta del héroe, $\downarrow$.

XXI persecución del héroe, Pr.

XXII socorro al héroe, Rs.

XXIV pretensión injustificada por el falso héroe, L.

XXV tarea difícil propuesta al héroe, $\mathrm{M}$.

XXVI tarea cumplida por el héroe, $\mathrm{N}$.

XXVIII descubrimiento del falso héroe o del agresor, Ex.

XXX castigo del falso héroe o del agresor, $\mathrm{U}$.

$X X X I$

matrimonio y ascenso al trono, $\mathrm{W}$.
} 
récit (1972) que puede servir como método de análisis, pero limitado a la narratología, por lo que prescinde de algunos aspectos importantes para la comparación exacta de relatos, como la estructura de estas narraciones o las figuras.

Ya en la antigüedad existía la fórmula prodesse et delectare como motivo central de la composición de los textos. Los hermanos Grimm y Cecilia Böhl de Faber indicaron que habían publicado sus colecciones con el mismo objetivo (Grimm 1986a: VIII y Amores 2001: 40). Hoy en día, mucha gente cree que los cuentos resultan demasiado crueles e inadecuados para el ánimo infantil (Grimm 1986a: VIIIs y Röhrich ${ }^{31974: ~ 46), ~}$ mientras que otros, por el contrario, destacan la importancia de los cuentos para la enseñanza y psicoterapia (Pöge-Alder ${ }^{2} 2011$ : 232-236) y añaden que se puede aprender mucho sobre etapas culturales olvidadas (Rodríguez Almodóvar 1988: 85) ${ }^{7}$.

Por tanto, para averiguar cuáles son las características comunes de los relatos de Fernán Caballero y de los hermanos Grimm, procedemos al análisis de las dos parejas ya mencionadas; y en primer lugar, los cuentos de animales.

El cuento "El lobo bobo y la zorra astuta" de Fernán Caballero y su versión alemana semejante "Katz" und Maus in Gesellschaft" de los hermanos Grimm trata de una zorra o un gato que traiciona a su amigo, bien sea el lobo o sea el ratón, inventando tres bautismos. En realidad, no van al bautismo, sino comen la miel del lobo o la grasa que sirve como acopio para el invierno. Al final, el lobo y el ratón descubren la traición, pero sin embargo el estafador, o bien el más fuerte, gana: un aspecto atípico para el género del cuento - esto es una característica del género de la fábula.

En otras versiones, en vez del lobo o del ratón actúa un chacal, que sustituye a la zorra o al gato, la miel o la grasa pueden ser mantequilla, y el bautizo está a veces sustituido por un funeral o una boda (Uther 2004: 26). Según la colección alemana con anotaciones a los Kinder- und Hausmärchen ya mencionada existe igualmente una versión de Basile de ese cuento de animales (Bolte-Polívka²1963a: 11) y según Friedrich von der Leyen, el cuento proviene probablemente de los países nórdicos (Von der Leyen 1964: 258).

Fernán Caballero utilizó en su versión una fórmula introductoria, mientras que la versión de los hermanos Grimm empieza con "Eine“ (la introducción con el artículo indeterminado se encuentra a menudo en los cuentos alemanes). En ambas versiones no existen fórmulas de cierre. Ni Fernán Caballero ni los hermanos Grimm jugaron con colores o números. Como en otros cuentos, están integradas típicas palabras y frases hechas que estructuran la trama (e.g. "Un día", "Pasado algún tiempo", "Einstmals", "Bald danach").

\footnotetext{
${ }^{7}$ Según Bruno Bettelheim, el aspecto psicológico del cuento resulta muy importante para la formación mental de un niño. Los cuentos ayudan al destinatario infantil a ordenar el caos de la vida, ofrecen oportunidades de identificación porque aparecen figuras malas y buenas, unidimensionales y parecidas al hombre. El niño puede vivir sus, aficiones malas' y aprender que nunca se debe perder la esperanza, porque una situación tiene un buen final (Bettelheim 1976: 5, 8s., 10, 40, 52, 74). Además, los niños entienden explicaciones fantásticas mejor que explicaciones realistas de los padres. Dos elementos claves de los cuentos son, por un lado, el animismo, es decir la opinión de niños que todo lo que se mueve, vive (Bettelheim 1976: 46ss.); y por otro lado, el tratamiento explícito de la muerte (Solms 1991: 212).
} 
La religión como elemento de la poesía natural está muy presente: la zorra y el gato son la madrina o el padrino de los bebés fingidos en el bautismo inventado. En la versión alemana, el ratón y su compañero el gato esconden el recipiente con grasa para el invierno bajo el altar en una iglesia porque les parece el lugar más seguro. Los niños en el bautismo español poseen nombres de santos, y el lenguaje recoge exclamaciones religiosas, además de una alusión al octavo mandamiento del Decálogo ("falso testimonio").

El lugar y el tiempo no están determinados de manera concreta. Sin embargo, la trama puede estar localizada en las viviendas de los animales y en una iglesia. Resulta obvio que pasan varios días. En la versión alemana se indica que el invierno llega pronto.

Si se analizan los cuentos con el método de Vladimir Propp, es posible hacer las series de funciones siguientes ${ }^{8}$ :

\section{El lobo bobo y la zorra astuta:$$
\mathbf{a}^{2} 3 \times\left\{\boldsymbol{\eta}^{1} \boldsymbol{\theta}^{1} \boldsymbol{\beta}^{1}\right\} Y \mathbf{H} \eta^{1} \theta^{10} \mathbf{J}
$$ \\ Katz' und Maus in Gesellschaft:$$
\mathbf{a}^{2} 3 x\left\{\eta^{1} \boldsymbol{\theta}^{1} \beta^{1}\right\} \beta^{1} \mathbf{H} \quad{ }^{\circ} \mathbf{J}
$$

Existe una situación inicial, en la que se introducen las figuras, y una situación de carencia $\left(\mathrm{a}^{2}\right)$, después se encuentra una triplicación representada por los tres engaños $\left(\eta^{1}\right)$. El lobo y el ratón dejan engañarse cada vez $\left(\theta^{1}\right)$ y la zorra y el gato salen tres veces de la casa para ir al bautismo fingido $\left(\beta^{1}\right)$. La invitación de la zorra no puede estar identificada como una función $(Y)$; en la versión alemana, el gato y el ratón salen juntos de la casa para ir a recoger el recipiente con la grasa $\left(\beta^{1}\right)$. En ambas narraciones está presentado un combate verbal $(\mathrm{H})$ entre las figuras. La escena con la prueba para saber quién ha engañado a quién - el culpable es quien suda la miel - solamente existe en la versión española $\left(\eta^{1} \theta^{1}\right)$ y representa un cuarto engaño. Ambos cuentos terminan con la función de la victoria sobre el agresor. No obstante, en estos dos casos resulta más bien la victoria del falso héroe o agresor sobre el héroe ( $\mathrm{o})$.

Como esferas de acción existen, por un lado, la esfera del héroe, representada hasta cierto punto por el ratón y el lobo, porque ellos son los buenos, y por otro lado, la esfera del agresor y del falso héroe, en la persona de la zorra o del gato. En la versión española aparecen además las dos zorritas - las niñas de la zorra - y el vendedor ambulante, que representan un tipo de ayudantes. El lobo compra la miel del vendedor y con ella se introduce el desencadenante de la traición. Las zorritas ofrecen el motivo perfecto para asegurarse de que el lobo sale de su casa. Además existen unas motivaciones especiales como la miel o la grasa para la zorra y el gato.

La versión del cuento de Fernán Caballero no parece cruel, sino resulta muy divertida en cuanto a la estupidez del lobo. En la versión de los hermanos Grimm, al contrario, existe un elemento brutal, porque el gato se traga al final a su propio compañero. Con los animales parlantes y humanizados se integra un componente maravilloso. El lector puede identificarse, o bien con el héroe, o bien con el falso héroe. En el segundo lugar, se experimentan sus malas aficiones. Ese tipo de cuento trata en general de la amistad, la lealtad y la confianza: tres buenas virtudes importantes.

\footnotetext{
${ }^{8}$ Se indica lo que coincide en ambas versiones.
} 
Ni Fernán Caballero ni los hermanos Grimm utilizaron en su narración versos y tampoco muchos recursos estilísticos. El único componente especial resulta probablemente de la coincidencia entre la acción de la zorra o del gato y el nombre del niño imaginado: la primera vez, cuando empieza a comer la miel o la grasa, el niño se llama "Empezili" o "Hautab"; la segunda vez el niño se llama "Mitadili" o "Halbaus" (porque la estafadora/el estafador come la mitad); y la tercera vez el niño se llama "Acabili" o "Ganzaus" (porque se come todo). Con respecto al tono infantil, este recurso está muy bien aplicado.

Los héroes y los falsos héroes no están caracterizados por muchos adjetivos o atributos. No obstante, sus caracteres obviamente están descritos por su comportamiento. El ratón es muy leal y un buen amigo, el gato está presentado con una gran voracidad, insidia y deslealtad. El lobo es - como ya se puede ver por el título - bobo y su compañera la zorra es exactamente el otro extremo, es decir astuta. Jacob Grimm describió las características típicas del zorro y del lobo en un capítulo de su obra Reinhart Fuchs ("Träger der Thierfabel", 1834). En esa obra la zorra es de color rojo y delgada, presenta una gran astucia y deslealtad, posee un carácter lisonjero y la función de una estafadora, pero en otros cuentos representa más bien un consejero por su gran experiencia (como en la fábula clásica) o como maestro. En cambio el lobo se presenta viejo, gris, gordo, tonto y torpe. En otros cuentos aparece cruel, codicioso, insaciable y voraz, y como ladrón o asesino (Grimm 1834: XXXIV). En la versión española se menciona otra figura importante de las fábulas: el león quien ejerce la función del juez en controversias (Grimm 1834: VII).

Las frases de ambas narraciones resultan un poco largas. Predomina el diálogo para que los cuentos parezcan animados y dinámicos. Se utiliza un lenguaje coloquial con palabras abreviadas e interjecciones típicas. Un último aspecto interesante en la narración de Fernán Caballero es el hecho de que utilizó la forma de tratamiento usted, que es inusual en los cuentos de hoy. En los otros cuentos de la coleccionista española casi solamente usa el tuteo.

En el cuento español se hallan dos motivos según el índex de Thompson: K372 "Playing Godfather" y K401.1 "Thief accuses his companion of having stolen the gold they have both stolen" a pesar de que el oro está sustituido por la miel que, en realidad, no han robado (Thompson 1957a: 284s. Y 285); en el cuento alemán se encuentra solamente el primer motivo.

En los cuentos de encantamiento "El lirio azul" y "Der singende Knochen", la temática es la siguiente: tres hermanos deben cumplir una tarea para el rey y el menor logra hacerlo. Sus hermanos mayores muy envidiosos lo matan y entierran. El mayor gana la mano de la princesa y el reino. Un día, un pastor pasa por la tumba y ve una cañita o un huesito. Coge la cañita o el huesito y fabrica un instrumento con el objeto. Cuando toca el instrumento, se descubre la verdad sobre el crimen. El rey manda desenterrar al hermano menor y castiga a los mayores.

Ya hemos indicado que este cuento posee asimismo, según Johannes Bolte y Georg Polívka, una relación de parentesco con una narración de Basile (Bolte-Polívka² 
1963a: 265). Probablemente proviene de Flandes y surgió a partir de una canción ${ }^{9}$, de la que existen varias versiones (con diferentes instrumentos: flauta, gaita, violín, arpa o cuerno) (Mackensen 1923: 3ss., 77 y Bolte-Polívka²1963a: 276). Las versiones españolas tienen influencia francesa, en las versiones alemanas pueden rastrearse contactos con tradiciones de varios países (Mackensen 1923: 84, 104).

Fernán Caballero recogió además otra versión del cuento, la que publicó en su obra Lágrimas. En esa versión la trama está narrada de manera un poco diferente: Los tres hermanos deben traer la flor Lililá a su padre, el rey. El menor logra resolver la tarea con la ayuda de la Virgen María y de unos niños blancos. Los hermanos lo matan y entierran. Un pastor fabrica una flauta de un cañaveral que sobresale de la tumba. El rey reconoce la voz que canta y condena a morir a sus otros hijos, pero, el niño muerto pide a su padre que muestra piedad, y el rey perdona a sus hijos (Caballero 1900: 57-60).

El título "El lirio azul" pone enfoque en el objeto deseado, el título “Der singende Knochen" en el instrumento. En el cuento alemán no existe ni una fórmula introductoria especial - empieza con "Ein", es decir, otra vez sólo el artículo indeterminado - ni una fórmula de cierre. La versión española al contrario empieza con la fórmula "Había un vez" y termina con la fórmula "Vivió y reinó muchos años, pero siempre sin un dedo. Cuento contado, ya se ha acabado, y por la chimenea se fue al terrado". Para estructurar la trama, Fernán Caballero y los hermanos Grimm usaron solamente unas pocas fórmulas épicas y algunas palabras típicas (e.g. "En esto pasó", "Fue tocando esto hasta", "Dann", "Also geschah es").

Se puede encontrar dos componentes religiosos en el cuento "Der singende Knochen". Por un lado el huesito está descrito como "blanco como la nieve“ y por el adjetivo "puro", dos características de la virginidad. Por otro lado, entierran al menor decorosamente en el cementerio de una iglesia.

En ambas narraciones, el lugar es más preciso que en la mayoría de los otros cuentos, sin embargo todavía se habla de un reino "por esos mundos", es decir, existente en cualquier lugar, sea donde sea. Otros lugares que aparecen en el cuento son el bosque - un lugar típico en cuentos alemanes -, el puente bajo el que está la tumba y una taberna. En la versión de Fernán Caballero falta una indicación exacta de tiempo. La versión alemana dice que los hermanos matan al menor por la tarde y que pasan varios años hasta que el pastor encuentra el huesito. En el personaje del pastor se hace presente un oficio tradicional, como suele suceder en los cuentos. El encuentro del huesito o de la cañita resulta un elemento romántico que representa el juicio de Dios, no escrito de la revelación de un asesinato de un inocente (Uther ${ }^{2} 2013:$ 74).

Analizando las funciones de estos dos cuentos con el método de Vladimir Propp, es posible hacer las series de las siguientes funciones:
El lirio azul:
$\mathrm{a}^{4} \mathbf{B}^{2} \mathrm{MC} \uparrow \quad \mathrm{GN} \downarrow \operatorname{Pr} \quad \mathbf{L K C F}^{3} \mathrm{ExJRs} \mathrm{UI}^{1} W^{\circ}$
Der singende Knochen: $\mathrm{AB}{ }^{2} \mathrm{MC} \uparrow \mathrm{EFGN} \downarrow \zeta^{1} \eta^{1} \theta^{1} \mathrm{HLKCF}^{3} \mathrm{ExJ} \quad \mathrm{U}$

\footnotetext{
${ }^{9}$ Existen canciones germánicas antiguas y romances escandinavos, ingleses y alemanes con la temática del cuento (Von der Leyen 1964: 67s.).
} 
El cuento español empieza con una situación de carencia $\left(\mathrm{a}^{4}\right)$, el cuento alemán con más o menos una situación en la que un antagonista (un jabalí) da perjuicios a una figura (A). Los monarcas de los cuentos prometen el reino y la mano de la princesa a la persona que logra cumplir una tarea $\left(\mathrm{B}^{2}\right)$ combinada con una prueba para el héroe (M). Los hermanos deciden actuar (C) y salen de la casa $(\uparrow)$.

El menor en la narración alemana encuentra en su viaje a un hombrecito en un bosque que le da un palo para matar un jabalí, aunque no se sabe por qué el hombre le da el objeto mágico al menor (EF). A continuación, los hermanos menores de ambas versiones llegan al lugar del objeto deseado $(G)$, es decir, pueden cumplir la tarea del rey $(\mathrm{N})$. Después emprenden el camino a casa $(\downarrow)$.

En la narración alemana está integrada además una escena en la que el menor encuentra a sus hermanos y les cuenta todo. Los hermanos planean matarlo. Desgraciadamente, el menor se deja engañar $\left(\zeta^{1} \eta^{1} \theta^{1}\right)$ y hasta cierto punto, esa escena representa un combate en el cuento de los hermanos Grimm $(\mathrm{H})$, aunque según Vladimir Propp, esta función se excluye con la pareja de funciones XXV y XXVI (tarea difícil) (Propp 1970: 124). En la narración de Fernán Caballero, los hermanos persiguen a su hermano menor (Pr). El mayor le dice al rey que él ha resuelto la tarea y disuelto la carencia o el perjuicio (LK). El pastor llega a la tumba y así empieza la acción opuesta, lo que resulta más o menos la función $X(C)$. El pastor fabrica el instrumento: un objeto mágico $\left(\mathrm{F}^{3}\right)$ con el que él revela la verdad (Ex). Al final, el menor gana sobre sus hermanos $(J)$ - en el cuento español además está vivo y salvado (Rs) - y el soberano ${ }^{10}$ manda a castigar a los hermanos mayores (U). En la narración de Fernán Caballero está indicado por último que al menor le falta un dedo, es decir está marcado $\left(\mathrm{I}^{1}\right)$ y que por fin gana la mano de la princesa y el trono $\left(\mathrm{W}_{\mathrm{o}}^{\mathrm{o}}\right)$.

Las figuras que aparecen son un rey (en la versión española resulta que además es el padre) e indirectamente su hija, la princesa. Actúan un héroe (primero en búsqueda, después sufriendo) y dos agresores, de los que el mayor representa igualmente un falso héroe. En el cuento alemán está presente asimismo un donante, en el personaje del hombrecito. En las dos versiones existe un ayudante parcial representado por el pastor.

Fernán Caballero y los hermanos Grimm integraron el aspecto de la triplicación en sus versiones porque se trata de tres hermanos: esto resulta una triplicación típica de cuentos. La melodía del instrumento ejerce la función de una cópula porque introduce la escena importante de la excavación.

En las dos narraciones se incorporan varios elementos crueles: el fratricidio descrito detalladamente en el cuento alemán, en el que además, se escava el esqueleto del menor. Al contrario de lo que sucede en al cuento de Fernán Caballero, los hermanos Grimm describieron cómo los hermanos sufren un castigo y mueren ahogados. Por último, existe una descripción brutal con el jabalí, que hace pedazos a todos.

Con este cuento los niños pueden vivir sentimientos malos como la envidia a un hermano o una hermana, lo que resulta un buen aspecto de cuentos conforme a

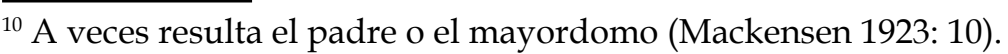


Bruno Bettelheim. Los componentes maravillosos son el instrumento, que resulta del mismo modo animado y en la versión de los hermanos Grimm existe además un objeto mágico que es el palo que el héroe obtiene del hombrecito, que forma parte del mundo maravilloso, porque representa seguramente al rey del bosque ${ }^{11}$.

Los tres coleccionistas incorporaron versos en sus narraciones refiriéndose a la canción del instrumento. En "El lirio azul“ los versos están escritos en la lengua valenciana. En el cuento alemán existen diversos diminutivos que suavizan los aspectos horripilantes y acercan el cuento al tono infantil. Los hermanos Grimm describieron a los tres hermanos con los típicos atributos: el mayor es astuto, el segundo es de inteligencia media, y el menor es inocente y tonto. Los hermanos de la versión de Fernán Caballero no están caracterizados especialmente.

La sintaxis resulta simple y predominan las frases paratácticas. Los apócopes y las formas apostrofadas aproximan el leguaje del cuento al lenguaje coloquial de la realidad extratextual. Por último, está presente incluso una lengua peninsular más en la versión española.

En ambas narraciones existen por lo menos tres motivos del índex de Stith Thompson: D1610.34 "Speaking musical instrument", E632 “Reincarnation as musical instrument" y N271 “Murder will out" (Thompson 1956a: 286, 489 y 1957b: 91). Si se considera la cañita no como dedo del héroe sino como una parte de una planta existen además los motivos D1610.2 "Speaking tree" (transformado en un instrumento) y E631 “Reincarnation in plant (tree) growing from grave“ (Thompson 1956a: 282 y 488). Otros motivos pueden resultar, por ejemplo, K2211 "Treacherous brother. Usually elder brother" y L0 “Victorious youngest child" o sea L10 "Victorious youngest son" (Thompson 1957a: 481 y 1957b: 6).

Existen otras seis parejas de cuentos emparentados que comparten - de la misma forma que las parejas analizadas antes en profundidad - la estructura y el contenido. En cuanto a los cuentos de encantamiento, hay que mencionar "BellaFlor" y "Ferenand getrü und Ferenand ungetrü" como dos cuentos que contienen algunos motivos que ya están integrados en la obra de Basile, de Madame d'Aulnoy y en la leyenda de Tristán e Isolda (Uther² 2013: 264 y Bolte-Polívka²1963c: 31); “Los deseos" y "Der Arme und der Reiche" que comparten el motivo de los tres deseos con diversas obras anteriores, entre ellas las obras de Charles Perrault y JeanneMarie Leprince de Beaumont, una fábula de Fedro, un lai de Marie de France o las "Metamorfosis" de Ovidio (Perrault-D’Aulnoy 1935: 61-64, Leprince de Beaumont 1995: 101-103, Grimm 1980: 160s., Bolte-Polívka² 1963b: 210-229 y Von der Leyen 1964: 285); y por último, “El duendecillo fraile" y "Von den Wichtelmännern" que pueden provenir de otra tradición folclórica de Alemania: la saga (Uther² 2013: 95). En cuanto a los cuentos de animales, resultan interesantes "El Carlanco" y "Der Wolf und die sieben jungen Geißlein“ que muestran motivos de una fábula de Jean de

\footnotetext{
${ }^{11}$ El rey del bosque es una figura de algunos cuentos que ayuda o impide a los cazadores matar un animal o despista al cazador. Normalmente el héroe obtiene un objeto mágico del rey del bosque después de resolver una tarea (Röhrich 1991: 30 y 41).
} 
La Fontaine, de una fábula de Marie de France o de obras aún más antiguas como Metamorfosis de Ovidio o la Ars poetica de Horacio (Grimm 1980: 27 y Bolte-Polívka² 1963a: 40s.); "Benibaire" y su equivalente "Herr Korbes" en el que, probablemente, el cuento español se funda, y que puede tener su origen en Asia (Amores 2001: 117 y Von der Leyen 1964: 250). Por último, “La hormiguita“ y "Läuschen und Flöhchen" con una temática bien extendida que ya aparece en una narración de Basile (BoltePolívka²1963a: 295).

Las series de funciones para estas seis parejas de cuentos son las siguentes:

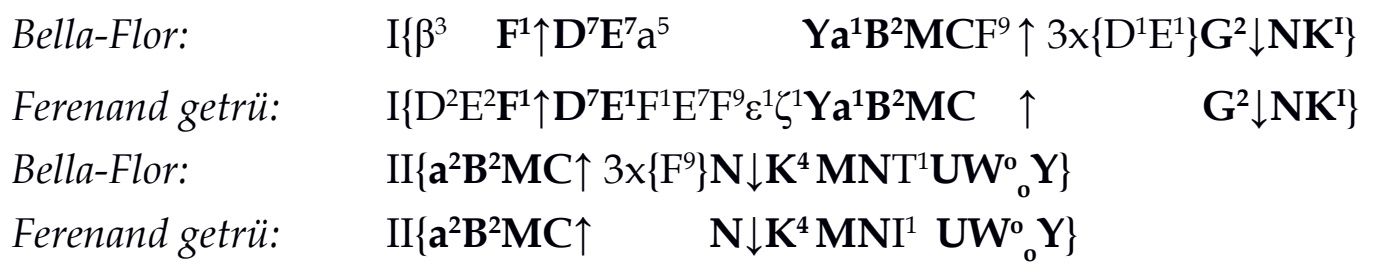

Los deseos: $\quad \mathrm{a}^{5} \mathrm{~F}^{1} \mathbf{D}^{7} \quad 2 \times\left\{\mathbf{E}^{1}\right\} \mathbf{A}^{6} \mathbf{K}^{4}$

Der Arme und der Reiche: $\quad \operatorname{I}\left\{\mathbf{D}^{2} 2 x\left\{\mathrm{E}^{2}\right\} \mathrm{F}^{1} \mathrm{~T}^{2}\right\} \operatorname{II}\left\{\beta^{1} \varepsilon^{1} \zeta^{1} \operatorname{Pr} L \gamma \delta \mathrm{F}^{1} \downarrow 2 x\left\{\mathbf{E}^{1}\right\} \mathbf{A}^{6} \mathbf{K}^{4} \mathrm{U}\right\}$

El duendecillo fraile: $\quad$ EF $\quad$ \&לExFMN

Von den Wichtelmännern: $\quad \mathrm{a}^{2} \mathbf{E F K}{ }^{4} \boldsymbol{\varepsilon} \zeta \mathbf{E x F M N}$

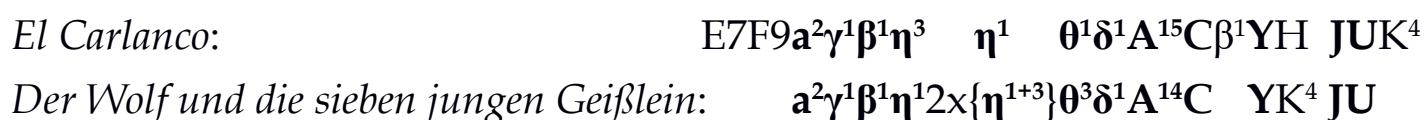

Benibaire: $\quad \mathrm{a}^{2 \uparrow} 5 \mathrm{x}\left\{\boldsymbol{\varepsilon} \zeta \mathbf{F}^{6}{ }_{9}\right\} \mathbf{G}^{2} \mathbf{Y H J W} \mathbf{W}_{\mathrm{o}}$

Herr Korbes: $\quad \uparrow \quad \boldsymbol{\varepsilon}_{\boldsymbol{\zeta}} \mathbf{F}^{6}{ }_{9} \mathbf{G}^{2} \mathbf{Y H J}$

La hormiguita: $\quad \mathrm{YWo} \beta^{1} \gamma \delta \mathbf{A} 5 \times\{\mathbf{Y}\}$

Läuschen und Flöhchen: $\quad \mathbf{A} 7 \mathbf{X}\{\mathbf{Y}\} \mathbf{Y}$

Se puede ver que, aparte de las parejas de "Los deseos" y "Der Arme und der Reiche" y "La hormiguita" y "Läuschen und Flöhchen", todas las otras parejas de cuentos - asimismo "El lobo bobo y la zorra astuta" y "Katz' und Maus in Gesellschaft" y "El lirio azul" y "Der singende Knochen" se parecen bastante en las funciones y de esa manera, en la estructura y el contenido.

Después del análisis en detalle, es posible deducir las siguientes conclusiones. En los cuentos de parentesco se observan dependencias e influencias entre las distintas versiones (de diferentes países) e incluso entre los distintos géneros narrativos de la tradición popular además de encontrarse varios motivos de obras de siglos pasados (e.g. de Charles Perrault: Contes du temps passé avec des Moralités, de Basile: Pentamerone, de Marie de France: Lais, del Panchatantra o de Apuleyo). 
El método de Vladimir Propp resulta siempre aplicable, pero solamente hasta cierto punto, con referencias no sólo a otros tipos de cuentos, como por ejemplo cuentos de animales, sino también a cuentos de encantamiento, es decir el tipo de cuento que sirve de fundamento a la teoría del estructuralista.

Con vistas a Bruno Bettelheim y su tesis psicológica, es posible examinar la presencia del animismo y de puntos de identificación. Siempre existe un componente maravilloso o sobrenatural, en los cuentos de animales, por ejemplo los animales que hablan. Además, se integran algunos elementos moralizantes y religiosos. Los cuentos de los hermanos Grimm resultan mucho más crueles que los cuentos de Fernán Caballero, que muestran más desenlaces felices y menos muertos que los cuentos alemanes. La coleccionista española tenía a primera vista la intención de publicar cuentos para un público infantil, los hermanos Grimm pensaban en un público adulto e infantil. A partir del momento en que solamente Wilhelm Grimm se dedicó a publicar las próximas ediciones de los dos volúmenes de los Kinderund Hausmärchen, cambió los cuentos adaptándolos a los niños, por ejemplo con la integración de versos o diminutivos. Con eso, Wilhelm Grimm cumplió el deseo de los lectores. Después de haber publicado la primera edición, Jacob se dedicó a otros proyectos.

En cada relato existen varios elementos típicos del género: figuras y lugares típicos, bodas reales y pruebas de fuego. Los cuentos españoles empiezan más a menudo con fórmulas introductorias y de cierre que las narraciones alemanas. Los lugares y el tiempo resultan siempre más bien indeterminados. La sintaxis está escrita de manera simple, se repiten ciertas palabras y fórmulas épicas, y el lenguaje se parece al lenguaje popular y coloquial del pueblo. Con una cantidad inmensa de discursos directos, los cuentos se aproximan a la vida real ${ }^{12}$. Con ellos, los coleccionistas lograron que los cuentos resultaran dinámicos y vivaces. Las figuras son de carácter simple y arquetípico, de lo que resulta otro elemento del romanticismo y de la tradición oral. Los relatos presentan a un buen héroe y a su malvado antagonista. Wilhelm Grimm y Fernán Caballero añadieron elementos humorísticos, recursos estilísticos y versos a los cuentos.

En total, con todas las modificaciones, muchos cuentos ya no parecen provenir de la tradición oral, sino más bien estar inventada, sin embargo, de todas maneras nunca han perdido su gran valor para niños y adultos por su función para el entretenimiento. Así lo demuestran todas las adaptaciones cinematográficas de hoy en día.

Concluimos este artículo con una fórmula de cierre típica:

$$
\begin{gathered}
\text { colorín, colorado, } \\
\text { este cuento se ha acabado, } \\
\text { pero si no dejamos de leer cuentos, } \\
\text { van a existir aún en futuros momentos. }
\end{gathered}
$$

\footnotetext{
${ }^{12}$ Algunos cuentos incluso están publicados en dialecto como por ejemplo el cuento alemán "Ferenand getrü un Ferenand ungetrü“.
} 


\section{BIBLIOGRAFÍA:}

\section{FUENTES PRIMARIAS}

Caballero, Fernán (1878): “Cuentos, oraciones, adivinas y refranes populares é infantiles", en: Colección de Autores Españoles, vol. XL, Leipzig: Brockhaus.

Encontrado en la página web: https://archive.org/details/cuentosoraciones00caba (consultado el 24 de septiembre de 2016)

Grimm, Jacob-Grimm, Wilhelm (1986a): Kinder- und Hausmärchen. Coleccionados por los hermanos Grimm (reimpresión de la primera edición de dos tomos de 1812 y 1815 basando en el ejemplar del Brüder-Grimm-Museum en Cassel con las diversas correcciones a mano y adiciones de los hermanos Grimm), vol. 1, Gotinga: Vandenhoeck \& Ruprecht.

Grimm, Jacob-Grimm, Wilhelm (1986b): Kinder- und Hausmärchen. Coleccionados por los hermanos Grimm (reimpresión de la primera edición de dos tomos de 1812 y 1815 basando en el ejemplar del Brüder-Grimm-Museum en Cassel con las diversas correcciones a mano y adiciones de los hermanos Grimm), vol. 2, Gotinga: Vandenhoeck \& Ruprecht.

\section{LITERATURA SECUNDARIA DEPENDIENTE E INDEPENDIENTE}

Amores, Montserrat (2001): Fernán Caballero y el cuento folclórico, El Puerto de Santa María: Bollullo Artes Gráficas.

Baquero Goyanes, Mariano (1992): El cuento español del Romanticismo al Realismo (edición revisada por Ana L. Baquero Escudero), Madrid: CSIC.

Basile, Giambattista (1954): Das Pentameron (traducido del italiano por Adolf Potthoff, con un epílogo de Benedetto Croce), Hattingen: Hundt-Verlag.

Bettelheim, Bruno (1976): The Uses of Enchantment. The Meaning and Importance of Fairy Tales, Londres: Thames and Hudson.

Bolte,Johannes-Polívka, Georg ( $\left.{ }^{2} 1963 a\right)$ :Anmerkungen zu den Kinder-und Hausmärchen der Brüder Grimm (N 1-60), vol. 1, Hildesheim: Georg Olms Verlagsbuchhandlung.

Bolte, Johannes-Polívka, Georg (21963b): Anmerkungen zu den Kinder- und Hausmärchen der Brüder Grimm ( $\mathrm{N}^{\circ}$ 61-120), vol. 2, Hildesheim: Georg Olms Verlagsbuchhandlung.

Bolte, Johannes-Polívka, Georg (21963c): Anmerkungen zu den Kinder- und Hausmärchen der Brüder Grimm (N 121-225), vol. 3, Hildesheim: Georg Olms Verlagsbuchhandlung.

Caballero, Fernán (1900): “Obras completas. Novelas. Lágrimas", vol. IV, en: Colección de Escritores castellanos. Novelistas, Madrid: Est. Tipográfico «Sucesores de Rivadeneyra». 
Faivre, Antoine (1979): Les contes de Grimm, mythe et initiation, Paris: Éditions Lettre Modernes.

Gerstner, Hermann (1952): Die Brüder Grimm. Ihr Leben und Werk in Selbstzeugnissen, Briefen und Aufzeichnungen, Ebenhausen: Wilhelm Langewiesche-Brandt.

Grimm, Jacob (1834): Reinhart Fuchs, Berlín: Reimer.

Encontrado en la página web: http:/ / books.google.de/books/about/Reinhart_ Fuchs.html? hl=de\&id=RQgYAAAAMAAJ (consultado el 24 de septiembre de 2016)

Grimm, Jacob-Grimm, Wilhelm (1980): Kinder- und Hausmärchen (con los comentarios originales de los hermanos Grimm y un anexo editado por Heinz Rölleke), vol. 3, Stuttgart: Philipp Reclam Jun.

Hamann, Hermann (1970) [1906]: Die literarischen Vorlagen der Kinder-und Hausmärchen und ihre Bearbeitung durch die Brüder Grimm, Nueva York: Johnson.

Herrero, Javier (1963): Fernán Caballero: un nuevo planteamiento, Madrid: Gredos.

Klibbe, Lawrence H. (1973): Fernán Caballero, Nueva York: Twayne Publishers, Inc.

Leprince de Beaumont, Jeanne-Marie (1995): Le magasin des enfants. La Belle et la Bête et autres contes, Arles: Éditions Philippe Picquier.

Lüthi, Max $\left(2004^{10}\right)$ : Märchen (elaborado por Heinz Rölleke), Stuttgart/Weimar: Metzler.

Mackensen, Lutz Dr. (1923): “Der singende Knochen. Ein Beitrag zur vergleichenden Märchenforschung", en: Walter Anderson et al. (eds.): FF Communications, $\mathrm{N}^{\circ}$ 49, Helsinki: Suomalainen Tiedeakatemia.

Morote Magán, Pascuala (2002): “El cuento de tradición oral y el cuento literario: de la narración a la lectura“", en: Antonio Mendoza Fillola (coord.): La seducción de la lectura en edades tempranas, Madrid: Ministerio de Educación, Cultura y Deporte, Secretaría General Técnica, 159-197.

Nathhorst, Bertel (1969): Formal or Structural Studies of Traditional Tales. The usefulness by Vladimir Propp, Alan Dundes, Claude Lévi-Strauss and Edmund Leach (tesis doctoral Universidad de Estocolmo), Estocolmo: Kungl Boktryckeriet P.A. Norstedt \& Söner.

Neumann, Siegfried (1986): “Zur Entstehung und zum Charakter der Grimmschen "Kinder- und Hausmärchen". Bemerkungen aus volkskundlicher Sicht", en: Prof. Dr. Heinz Stiller (ed.): Jacob und Wilhelm Grimm. Vorträge anläßlich der 200. Wiederkehrihrer Geburtstage (4. Januar 1785/24. Februar 1786), Sitzungsberichte der Akademie der Wissenschaften der DDR, Año 1985, Nº 6/G, Berlín: AkademieVerlag, 55-64.

Perrault, Charles-D'Aulnoy, Marie-Catherine (1935): Contes en vers et en prose, París: Ernest Flammarion. 
Pöge-Alder, Kathrin (20112): Märchenforschung. Theorien, Methoden, Interpretationen, Tubinga: Narr Verlag.

Propp, Vladimir (1970): Morphologie du conte suivi de Les transformations des contes merveilleux et de E. Mélétinski L'étude structurale et typologie du conte (traductions de Marguerite Derrida, Tzvetan Todorov et Claude Kahn), París: Éditions du Seuil.

Rodríguez Almodóvar, Antonio (1988): “Los cuentos populares o la tentativa de un texto infinito“, en: José Varela Ortega (dir.): Revista de Occidente, N 91, Madrid: Fundación José Ortega y Gasset-Gregorio Marañón, 81-93.

Röhrich, Lutz (1974³): Märchen und Wirklichkeit, Wiesbaden: Franz Steiner.

Röhrich, Lutz (1991): “Der Herr der Tiere", en: Arnica Esterl y Wilhelm Solms (eds.): Tiere und Tiergestaltige im Märchen, Regensburg: Erich Röth Verlag, 30-47.

Rölleke, Heinz (1985): “Die Märchen der Brüder Grimm. Eine Einführung“, en: Peter Brang et al. (eds.): Artemis Einführungen, vol. 18, Múnich-Zúrich: Artemis Verlag. Schlegel, August Wilhelm (1965): Geschichte der romantischen Literatur, Stuttgart: W. Kohlhammer Verlag.

Schlegel, August Wilhelm (1966): "Vorlesungen über dramatische Kunst und Literatur", vol. 1, en: Edgar Lohner (ed.): Kritische Schriften und Briefe, V, Stuttgart/Berlín/ Colonia/Maguncia: W. Kohlhammer Verlag.

Solms, Wilhelm (1991): "Die Gattung Grimms Tiermärchen", en: Arnica Esterl y Wilhelm Solms (eds.): Tiere und Tiergestaltige im Märchen, Regensburg: Erich Röth Verlag, 195-215.

Thompson, Stith (1956a): Motif-Index of Folk-Literature. A Classification of Narrative Elements in Folktales, Ballads, Myths, Fables, Mediaval Romances, Exempla, Fabliaux, Jest-Books and Local Legends (revidaso y ampliado), vol. 2, Copenhague: Rosenkilde and Bagger.

Thompson, Stith (1957a): Motif-Index of Folk-Literature. A Classification of Narrative Elements in Folktales, Ballads, Myths, Fables, Mediaval Romances, Exempla, Fabliaux, Jest-Books and Local Legends (revidaso y ampliado), vol. 4, Copenhague: Rosenkilde and Bagger.

Thompson, Stith (1957b): Motif-Index of Folk-Literature. A Classification of Narrative Elements in Folktales, Ballads, Myths, Fables, Mediaval Romances, Exempla, Fabliaux, Jest-Books and Local Legends (revidaso y ampliado), vol. 5, Copenhague: Rosenkilde and Bagger.

Uther, Hans-Jörg (2004): “The Types of International Folktales. A Classification and Bibliography" (Part I, Animal Tales, Tales of Magic, Religious Tales, and Realistic Tales), en: Satu Apo et al. (eds.): FF Communications, $\mathrm{N}^{\circ} 284$, Helsinki: Suomalainen Tiedeakatemia. 
Uther, Hans-Jörg (2013²): Handbuch zu den »Kinder- und Hausmärchen « der Brüder Grimm. Entstehung - Wirkung - Interpretation, Berlín-Boston: De Gruyter.

Von Beit, Hedwig (1952): Symbolik des Märchens. Versucheiner Deutung, Bern: A. Francke AG.

Von der Leyen, Friedrich (1964): Das deutsche Märchen und die Brüder Grimm, Düsseldorf/ Colonia: Eugen Diederichs. 